\title{
Polarization of Macrophage and Lupus Nephritis
}

\author{
Qiongyi Hu and Qiang Wang* \\ Department of Dermatology, Zhongshan Hospital, Fudan University, Shanghai, PR China
}

*Corresponding author: Qiang Wang, Department of Dermatology, Zhongshan Hospital, Fudan University, China, E-mail: wang.qiang@zs-hospital.sh.cn

Received date: January 4, 2016; Accepted date: January 7, 2016; Published date: January 25, 2016

Copyright: (c) $2016 \mathrm{Hu}$ and Wang. This is an open-access article distributed under the terms of the Creative Commons Attribution License, which permits unrestricted use, distribution, and reproduction in any medium, provided the original author and source are credited.

\begin{abstract}
Lupus nephritis ( $L N)$ is one of the common and severe complications in systemic lupus erythematosus (SLE), and is a leading cause of morbidity and mortality for SLE patients. It is well known that LN is characterized by the inflammation mediated by immune response in the kidney tissues, during which macrophage (Mø) plays a vital role. According to the varied microenvironments in different stages of lupus nephritis, macrophages are divided into two categories, namely classically activated macrophages (M1) and alternatively activated macrophages (M2). Macrophages can undergo phenotypical/functional switch and play different functions depending on multiple signal pathways, including STAT transcription factors, epigenetic aspects, NF-KB pathways, IRF transcription factors and some interleukins, chemokines and its receptors. Due to the heterogeneity and plasticity, the polarization of macrophages may exert an influence on the outcome of lupus nephritis. Thus, targeting the polarization of macrophages properly may become a new therapeutic treatment for lupus nephritis. This review focuses on how polarization of macrophages regulates the pathogenesis of $L N$.
\end{abstract}

\section{Key words:}

Lupus nephritis; Macrophages; Polarization; Inflammation

\section{Introduction}

Systemic lupus nephritis (SLE) is autoimmune disease that affects mainly women of reproductive age and which can affect multiple different organ systems. Kidney involvement, known as Lupus nephritis (LN), is an end organ manifestation seen in upwards of $60 \%$ of SLE patients. LN is a serious complication of SLE, and is a leading cause of morbidity and mortality for SLE patients [1]. Many studies implicate macrophage $(\mathrm{M} \varnothing)$ in the pathogenesis of $\mathrm{LN}$, although the exact nature of their contribution has not yet been elucidated. LN is chronic inflammatory disease with macrophages playing an important role [2]. Macrophages, as a kind of crucial immunocytes, mediate the initiation and progression of renal injury in various aspects. Macrophages induce the production of autoantibodies and also product pro-inflammatory cytokines, leading to injure the glomeruli and proteinuria onset [3]. Recently, much attention has been drawn to the relation between polarization of macrophages and LN. In the review, we mainly focus on how polarization of macrophages regulates the pathogenesis of LN.

\section{Macrophages and LN}

Heterogeneity and plasticity are hallmarks of macrophages, which drive from mononuclear phagocytes in blood system and differentiate into mature ones in peripheral tissue. Macrophages are important regulators of innate and adaptive immunity, as well as systemic metabolism, hematopoiesis, vasculogenesis and reproduction [4]. Macrophage differentiation refers to the process that cells migrate to the vessel wall from peripheral blood and then into the organizations, in which adhesion molecules, chemokines and cytokines will affect the migration of monocytes and late maturation [5], and polarization of macrophages refers to the phenotypical/functional switch due to macrophages completely differentiated in a particular tissue responding to the external stimuli. According to the varied microenvironments in different stages of lupus nephritis, macrophages are divided into two categories, namely classically activated macrophages (M1) and alternatively activated macrophages (M2) [6]. The dynamic balance between M1 and M2 in renal tissue is regulated by the microenvironments in different stages of $\mathrm{LN}$, which determine the progress and prognosis of LN. M1 macrophages initially enter the kidneys following acute renal injury and secrete pro-inflammatory cytokines [7-9]. In contrast, during the repair phase, these cells may switch their phenotype to a "M2" phenotype contributing to repair of epithelial cells and vascular endothelial cells and thus turn into renal fibrosis, correlating with poor outcome of $\operatorname{LN}[10,11]$.

\section{Classical Activated M1 Macrophages in LN}

The classical activation of macrophages is driven by the stimulation, such as IFN- $\gamma$, lipopolysaccharide (LPS), TNF- $\alpha$ and granulocyte macrophage colony- stimulating factor (GM-CSF), with an IL-12 ${ }^{\text {high }}$, IL-23 high, IL-10 low phenotype [12]. In general, M1macrophages contribute to the inflammatory response by the secretion of proinflammatory cytokines such as interleukin-6 (IL-6), IL-1 $\beta$, TNF, as well as IL-12 and IL-23 [13]. M1 macrophages also produce iuducible nitric oxide synthase (iNOS) as an important factor in response of the attack by bacterial, fungal, and viral infections [14,15]. Some types of chemokines receptor ligands, such as CXCL9 and CXCL10 expressed by M1 macrophages mediate polarization Th1 responses [16,17]. It is suggested that phagocytosis of tissue debris may switch Mø from proinflammatory to anti-inflammatory $[18,19]$. However, increased apoptosis and impaired clearance of apoptosis cells by macrophages have found in SLE patients and mice [20-24]. And recently, researches have reported that the dominance of M1 macrophages in kidney failed to skew towards M2 macrophages in MRL-Fas ${ }^{\text {pr }}$ and SLE-123 mice after $\mathrm{I} / \mathrm{R}$ and in spontaneous LN in MRL-Fas ${ }^{\mathrm{lpr}}$ mice by inducing CSF-1 in injured TEC, playing a central role in defective renal repair and non-resolving inflammation which lead the onset of LN [25]. 
Laquinimod is an immunomodulatory drug that has reduced the number of M1 macrophages, inhibited the secretion of TNF- $\alpha$, and increased the expression of IL-10 in both kidneys and spleen of (NZB $\times$ NZW) F1 mice, indicating that Laquinimod by inducing M1 macrophages shifting to M2 macrophages thus alleviated the progression of LN [26].

\section{Alternatively Activated M2 Macrophages in LN}

Markedly different from M1 macrophages, studies have divided alternatively activated M2 macrophages into M2a, M2b, and M2c. Of these, M2a macrophages induced by IL- 4 and IL-13 are capable of producing TGF- $\beta$ and arginase, as well as their ability to produce certain ECM components that play a role in tissue-repair; M2b macrophages, which are induced by TLR ligation or immune complexes, promote the production of IL-10 and decreases production of IL-12, exerting potent anti-inflammatory properties; M2c macrophages induced by IL-10 and glucocorticoids have a antiinflammatory role and deactivating M1 macrophages [27,28]. In an induced model of LN induced by activated lymphocytes derived DNA (ALD-DNA), intra-renal Mø polarize Mø toward an M2b phenotype [29]. In contrast, LN patients with both anti-dsDNA antibody and anti-Ro60 antibody positivity in serum showed significantly high levels of CD163 and Mer, which are M2c macrophages markers, and these correlate with SLEDAI [30]. Exposure to helminth immunomodulator, ES-62, secreted by the filarial nematode for two weeks, reduced deposition of IgG and C3 in the kidneys and increased the infiltrating of anti-inflammatory $\mathrm{F} 4 / 80^{\mathrm{hi}} \mathrm{CD} 11 \mathrm{c}^{-} \mathrm{CD} 206^{+} \mathrm{Ly}_{6 \mathrm{G}}{ }^{+} \mathrm{M} 2$ macrophages and afforded protection against pathology in the MRL/Lpr mouse model of SLE [31]. In addition, the chemokine receptor 1 inhibitor ameliorated glomerular and tubular injuries, delayed proteinuria by decreasing kidney accumulation of both M1 and M2 macrophages [32]. As lupus nephritis may be triggered by a diversity of mechanisms, it is reasonable that varied macrophages phenotypes reflect differences within patient populations with distinct mechanisms.

\section{Regulatory Factors of Macrophage Polarization and Therapeutic Targets in LN}

Macrophages are closely related to the pathogenesis of LN, so recently, much importance has been attached to heterogeneity and plasticity of macrophage polarization and the regulatory factors. Numerous lines of evidence suggest that macrophage polarization is associated with some signalling pathways, transcription factors, noncoding RNA, epigenetic and some interleukins (eg. MCP-1, CSF-1), chemokines and its receptors (eg. CX3CL, CX3CR), etc. Research on these regulatory mechanisms has been deep into the genetic level, thus providing important implications for targeted LN therapies.

\section{Signal Transducers and Activators of Transcription}

The signal transducers and activators of transcription (STAT) regulates many aspects of growth, survival and differentiation in cells and activated by Janus kinase (JAK). Transcription factors STAT1 activation promotes M1, whereas STAT3/6 activation promotes M2 macrophage polarization [33]. IFN- $\gamma$ can activate the phosphorylation STAT-1, leading to M1 macrophages activation; STAT-3, plays an important role in the inflammatory response by regulating inflammation-related proteins, can promote M2 macrophages activation in response to IL-10; IL-4 and IL-10 promote M2 macrophages associated proteins, arginase-1 (Arg-1), with the help of
STAT-6 [34]. Studies have demonstrated that total amount of STAT-3 and the activity was higher in bone marrow-derived mononuclear cells in patients with SLE than that in healthy patients. Inhibition of STAT-3 in LN mice leads to diminish the total number of T cells, weakens their capability of migration and also reduces antibody production [35].

Inhibitors of JAK/STAT pathway are currently in clinical trials for treating rheumatoid arthritis [36]. Therefore, inhibitors of JAK/STAT pathway are a promising immunomodulatory therapeutic agent for use in human LN.

\section{Interferon Regulatory Factor}

Interferon regulatory factor (IRF) is a class of transcription factor family regulating host defense and composed in humans of 9 distinct proteins. Besides to autoantibodies, the high prevalence of type I interferon (IFN-I) in serum is another hallmark of SLE [37]. Researchers have identified the IFN-I master regulator gene IRF7 is only hypomethylated in lupus patients with renal involvement. IRF-7 is an upstream transcription factor that regulates several loci demethylated only with renal involvement, such as CD80, interferoninducible protein 44 (IFI44), interferon-stimulated gene15 (ISG15), ISG20, integrins $\alpha \mathrm{X}$ (ITGAX) and poly ADP-ribose polymerase 12 (PARP12) [38]. In addition, IRF5 mediates the secretion of proinflammatory cytokines relating to SLE by Toll-like receptor (TLR), such as IL-6, IL-12, IL-17, IL-23 and TNF- $\alpha$ [39]. IRF not only can regulate the expression of IFN- $\alpha$, but also plays an important role in macrophage polarization. IRF3, IRF5 promote the M1 macrophages polarization, and IRF4 promote M2 polarized macrophages [40]. As a target for treatment of LN, regulation of IRF switch a new state of equilibrium for $\mathrm{M} 1$ and M2, thus alleviating the pathological progression of LN.

\section{Micro-RNA}

MicroRNAs (miRNAs) are a family of regulatory RNA molecules, similar to endogenous mediators of RNA interference that modulate apoptosis, differentiation, and activation of immune cells. It's indicated that there are six kinds of miRNAs dysfunction in peripheral blood mononuclear cells of SLE patients, including miRNA-21, miRNA-25, miRNA-125a, miRNA-146a, miRNA-148 and miRNA-186 that are known to regulate the immune and inflammation [41]. Banerjee et al [42] found that, miRNA-125a-5p regulated polarization of macrophages by Toll-like receptor. In miRNA-125a-5p deficient mice, the expression of TNF- $\alpha$, IL-12 and iNOS by M1 increased, and the expression of Arg-1 by M2 decreased, in contrast to over-expression of miRNA-125a-5p. According to discovering the experiment, Krüppellike factor 33 (KLF33) is the target gene of miRNA-125a-5p, thus regulating macrophage polarization. There are other miRNAs, such as miRNA-17, miRNA-20a, and miRNA-106a that regulate macrophage function with signal-regulated protein $\alpha$ as common target genes, but the mechanism remains for further study [43]. Thus, regulation of macrophage polarization by miRNAs may also provide another treatment for LN.

\section{Nuclear Factor- $\kappa B$}

Nuclear factor- $\kappa \mathrm{B}$ (NF- $\kappa \mathrm{B})$, a class of multi-effect of nuclear transcription factor, regulates the expression of many inflammatory genes in response to various physiological and environmental stimuli [44]. Studies on human LN have shown the increased activation of NF$\kappa \mathrm{B}$ pathway, as well as increased expression of TNF- $\alpha, \mathrm{IL}-1 \beta, \mathrm{IL}-6$ and 
intercellular adhesion molecule 1(ICAM-1), the downstream factor of classical activated NF- $\kappa B$ pathway $[45,46]$. TNF- $\alpha$ is the critical proinflammatory cytokine produced primarily by macrophages upon injury, and then stimulates the activation of classical pathway of NF$\kappa \mathrm{B}$, which in turn regulates the production of TNF- $\alpha$ [47,48]. Derived from monocytes and macrophages, IL-6 in the areas of inflammation release chemokines by stimulating endothelial cells to promote leukocyte infiltration and monocyte/macrophage migration [49]. In all patients with LN in kidney tissue in line with WHO standards, it is found that a kind of high mobility group protein (HMGB1) distributes along the glomerular epithelial and mesangial [50], such protein stabilizes nucleosome structure, but plays a pro-inflammatory role when released from the nucleus, mainly to promote the maturation of dendritic cells, macrophages promote pro-inflammatory cytokine release. By activating the NF- $\mathrm{KB}$ pathway, HMGB1 regulate the transcription of cell proliferation related proteins D1, promote mesangial cell proliferation, and thus participate in the pathogenesis of $\mathrm{LN}$ [51]. NF- $\kappa \mathrm{B}$ activation in glomeruli is related to SLE activity index and the levels of macrophage infiltration. Numerous reports found that NF- $\kappa \mathrm{B}$ p65/p50 promoted Mø to polarize to M1 [52]. Mesenchymal stem cells, with anti-inflammatory and immunosuppressive effects, found to relieve kidney inflammation in LN mice by increasing the number of M2 macrophages and their phagocytic ability [53] Moreover, bone marrow-derived mesenchymal stem cells promote M2 macrophages polarization by inhibiting NF- $\kappa B$ pathway [54]. Lately, our study suggests that treated by Demethylzeylasteral (T-96), one of triterpenoids isolated from the root xylem of Tripterygium wilfordii Hook F, MRL/lpr mice showed a significant improvement in $24 \mathrm{~h}$ proteinuria and the levels of anti-ds DNA antibody in serum. Additionally, renal pathological lesions were attenuated, which mainly attributed to reduced secretion of IL- 23 and TNF- $\alpha$ by M1 and inhibition of NF-kB pathway [55]. Thus, by selective inhibition of NF$\kappa \mathrm{B}$ pathway to further regulate macrophages polarization; it can be used as a target for the treatment of LN.

\section{Conclusion}

In summary, $\mathrm{LN}$ is characterized by inflammation reaction at early stage and fibrosis in later stage. LN is a severe complication of SLE that greatly diminishes patient quality of life and negatively affects survival. Highly specific treatment methods are lacking, with the primary clinical option continuing to the use of broadly immunosuppressive drugs. The macrophages in $\mathrm{LN}$ at different stages regulated by a variety of signalling pathways undergo a dynamic balance and closely related to the prognosis, which is closely related to the progress and prognosis of LN. Due to macrophages plasticity, not only unpolarized macrophages will switch to a certain phenotype, but also polarized M1/M2 macrophages will shift to another phenotype in response to microenvironments. Therefore, some agents or drugs by reducing the number of macrophages or altering their phenotype in the LN therapy will be possible as a new target for the treatment of LN.

\section{References}

1. Mohan C, Putterman C (2015) Genetics and pathogenesis of systemic lupus erythematosus and lupus nephritis. Nat Rev Nephrol 11: 329-341.

2. Davidson A, Aranow C (2006) Pathogenesis and treatment of systemic lupus erythematosus nephritis. Curr Opin Rheumatol 18: 468-475.

3. Sahu R, Bethunaickan R, Singh S, Davidson A (2014) Structure and function of renal macrophages and dendritic cells from lupus-prone mice. Arthritis Rheumatol 66: 1596-1607.
4. Tugal D, Liao X, Jain MK (2013) Transcriptional control of macrophage polarization. Arterioscler Thromb Vasc Biol 33: 1135-1144.

5. Martinez FO, Sica A, Mantovani A, Locati M (2008) Macrophage activation and polarization. Front Biosci 13: 453-461.

6. Schwartz YSh, Svistelnik AV (2012) Functional phenotypes of macrophages and the M1-M2 polarization concept. Part I. Proinflammatory phenotype. Biochemistry (Mosc) 77: 246-260.

7. Guo S, Wietecha TA, Hudkins KL, Kida Y, Spencer MW, et al. (2011) Macrophages are essential contributors to kidney injury in murine cryoglobulinemic membranoproliferative glomerulonephritis. Kidney Int 80: 946-958.

8. Anders HJ, Ryu M (2011) Renal microenvironments and macrophage phenotypes determine progression or resolution of renal inflammation and fibrosis. Kidney Int 80: 915-925.

9. Arnold CE, Whyte CS, Gordon P, Barker RN, Rees AJ, et al. (2014) A critical role for suppressor of cytokine signalling 3 in promoting M1 macrophage activation and function in vitro and in vivo. Immunology 141: 96-110.

10. Lee S, Huen S, Nishio H, Nishio S, Lee HK, et al. (2011) Distinct macrophage phenotypes contribute to kidney injury and repair. J Am Soc Nephrol 22: 317-326.

11. Filardy AA, Pires DR, Nunes MP, Takiya CM, Freire-de-Lima CG, et al. (2010) Proinflammatory clearance of apoptotic neutrophils induces an IL-12(low)IL-10(high) regulatory phenotype in macrophages. J Immunol 185: 2044-2050.

12. Mantovani A, Biswas SK, Galdiero MR, Sica A, Locati M (2013) Macrophage plasticity and polarization in tissue repair and remodelling. J Pathol 229: 176-185.

13. Colin S, Chinetti-Gbaguidi G, Staels B (2014) Macrophage phenotypes in atherosclerosis. Immunol Rev 262: 153-166.

14. Stein M, Keshav S, Harris N, Gordon S (1992) Interleukin 4 potently enhances murine macrophage mannose receptor activity: a marker of alternative immunologic macrophage activation. J Exp Med 176: 287-292.

15. Verreck FA, de Boer T, Langenberg DM, Hoeve MA, Kramer M, et al. (2004) Human IL-23-producing type 1 macrophages promote but IL-10producing type 2 macrophages subvert immunity to (myco)bacteria. Proc Natl Acad Sci U S A 101: 4560-4565.

16. Mantovani A (2008) From phagocyte diversity and activation to probiotics: back to Metchnikoff. Eur J Immunol 38: 3269-3273.

17. Martinez FO, Gordon S, Locati M, Mantovani A (2006) Transcriptional profiling of the human monocyte-to-macrophage differentiation and polarization: new molecules and patterns of gene expression. J Immunol 177: 7303-7311.

18. Fadok VA, Bratton DL, Konowal A, Freed PW, Westcott JY, et al. (1998) Macrophages that have ingested apoptotic cells in vitro inhibit proinflammatory cytokine production through autocrine/ paracrine mechanisms involving TGF-beta, PGE2, and PAF. J Clin Invest 101: 890-898.

19. Fadok VA, Bratton DL, Guthrie L, Henson PM (2001) Differential effects of apoptotic versus lysed cells on macrophage production of cytokines: role of proteases. J Immunol 166: 6847-6854.

20. Ren Y, Tang J, Mok MY, Chan AW, Wu A, et al. (2003) Increased apoptotic neutrophils and macrophages and impaired macrophage phagocytic clearance of apoptotic neutrophils in systemic lupus erythematosus. Arthritis Rheum 48: 2888-2897.

21. Baumann I, Kolowos W, Voll RE, Manger B, Gaipl U, et al. (2002) Impaired uptake of apoptotic cells into tingible body macrophages in germinal centers of patients with systemic lupus erythematosus. Arthritis Rheum 46: 191-201.

22. Herrmann M, Voll RE, Zoller OM, Hagenhofer M, Ponner BB, et al. (1998) Impaired phagocytosis of apoptotic cell material by monocytederived macrophages from patients with systemic lupus erythematosus. Arthritis Rheum 41: 1241-1250.

23. Tas SW, Quartier P, Botto M, Fossati-Jimack L (2006) Macrophages from patients with SLE and rheumatoid arthritis have defective adhesion in 
vitro, while only SLE macrophages have impaired uptake of apoptotic cells. Ann Rheum Dis 65: 216-221.

24. Potter PK, Cortes-Hernandez J, Quartier P, Botto M, Walport MJ (2003) Lupus-prone mice have an abnormal response to thioglycolate and an impaired clearance of apoptotic cells. J Immunol 170: 3223-3232.

25. Iwata Y, Boström EA, Menke J, Rabacal WA, Morel L, et al. (2012) Aberrant macrophages mediate defective kidney repair that triggers nephritis in lupus-susceptible mice. J Immunol 188: 4568-4580.

26. Lourenço EV, Wong M, Hahn BH, Palma-Diaz MF, Skaggs BJ (2014) Laquinimod delays and suppresses nephritis in lupus-prone mice and affects both myeloid and lymphoid immune cells. Arthritis Rheumatol 66: 674-685.

27. Novak ML, Koh TJ (2013) Macrophage phenotypes during tissue repair. J Leukoc Biol 93: 875-881.

28. Kharraz Y, Guerra J, Mann CJ, Serrano AL, Muñoz-Cánoves P (2013) Macrophage plasticity and the role of inflammation in skeletal muscle repair. Mediators Inflamm 2013: 491497.

29. Cai Y, Zhang W, Xiong S (2013) Mannose-binding lectin blunts macrophage polarization and ameliorates lupus nephritis. PLoS One 8 e62465.

30. Zizzo G, Guerrieri J, Dittman LM, Merrill JT, Cohen PL (2013) Circulating levels of soluble MER in lupus reflect M2c activation of monocytes/macrophages, autoantibody specificities and disease activity. Arthritis Res Ther 15: R212.

31. Rodgers DT, McGrath MA, Pineda MA, Al-Riyami L, Rzepecka J, et al. (2015) The parasitic worm product ES-62 targets myeloid differentiation factor 88-dependent effector mechanisms to suppress antinuclear antibody production and proteinuria in MRL/lpr mice. Arthritis Rheumatol 67: 1023-1035.

32. Bignon A, Gaudin F, Hémon P, Tharinger H, Mayol K, et al. (2014) CCR1 inhibition ameliorates the progression of lupus nephritis in NZB/W mice. J Immunol 192: 886-896.

33. Zhou D, Huang C, Lin Z, Zhan S, Kong L, et al. (2014) Macrophage polarization and function with emphasis on the evolving roles of coordinated regulation of cellular signaling pathways. Cell Signal 26: 192-197.

34. Motwani MP, Gilroy DW (2015) Macrophage development and polarization in chronic inflammation. Semin Immunol 27: 257-266.

35. Edwards LJ, Mizui M, Kyttaris V (2015) Signal transducer and activator of transcription (STAT) 3 inhibition delays the onset of lupus nephritis in MRL/lpr mice. Clin Immunol 158: 221-230.

36. Kyttaris VC (2012) Kinase inhibitors: a new class of antirheumatic drugs. Drug Des Devel Ther 6: 245-250.

37. Weckerle CE, Franek BS, Kelly JA, Kumabe M, Mikolaitis RA, et al. (2011) Network analysis of associations between serum interferon-Îे activity, autoantibodies, and clinical features in systemic lupus erythematosus. Arthritis Rheum 63: 1044-1053.

38. Coit P, Renauer P, Jeffries MA, Merrill JT, McCune WJ, et al. (2015) Renal involvement in lupus is characterized by unique DNA methylation changes in naïve CD4+ T cells. J Autoimmun 61: 29-35.

39. Lazzari E, Jefferies CA (2014) IRF5-mediated signaling and implications for SLE. Clin Immunol 153: 343-352.
40. Wang N, Liang H, Zen K (2014) Molecular mechanisms that influence the macrophage $\mathrm{m} 1-\mathrm{m} 2$ polarization balance. Front Immunol 5: 614 .

41. Picascia A, Grimaldi V, Pignalosa O, De Pascale MR, Schiano C, et al. (2015) Epigenetic control of autoimmune diseases: from bench to bedside. Clin Immunol 157: 1-15.

42. Banerjee S, Cui H, Xie N, Tan Z, Yang S, et al. (2013) miR-125a-5p regulates differential activation of macrophages and inflammation. J Biol Chem 288: 35428-35436.

43. Zhu D, Pan C, Li L, Bian Z, Lv Z, et al. (2013) MicroRNA-17/20a/106a modulate macrophage inflammatory responses through targeting signalregulatory protein Ît. J Allergy Clin Immunol 132: 426-436.

44. Wong ET, Tergaonkar V (2009) Roles of NF-kappaB in health and disease: mechanisms and therapeutic potential. Clin Sci (Lond) 116: $451-465$.

45. Zheng L, Sinniah R, Hsu SI (2006) In situ glomerular expression of activated NF-kappaB in human lupus nephritis and other nonproliferative proteinuric glomerulopathy. Virchows Arch 448: 172-183.

46. Zheng L, Sinniah R, Hsu SI (2008) Pathogenic role of NF-kappaB activation in tubulointerstitial inflammatory lesions in human lupus nephritis. J Histochem Cytochem 56: 517-529.

47. Ghosh S, Hayden MS (2012) Celebrating 25 years of NF-1 $\hat{I}^{\circ} B$ research. Immunol Rev 246: 5-13.

48. Parameswaran N, Patial S (2010) Tumor necrosis factor-ÎI signaling in macrophages. Crit Rev Eukaryot Gene Expr 20: 87-103.

49. Cash H, Relle M, Menke J, Brochhausen C, Jones SA, et al. (2010) Interleukin 6 (IL-6) deficiency delays lupus nephritis in MRL-Faslpr mice: the IL-6 pathway as a new therapeutic target in treatment of autoimmune kidney disease in systemic lupus erythematosus. J Rheumatol 37: 60-70.

50. Zickert A, Palmblad K, Sundelin B, Chavan S, Tracey KJ, et al. (2012) Renal expression and serum levels of high mobility group box 1 protein in lupus nephritis. Arthritis Res Ther 14: R36.

51. Feng X, Hao J, Liu Q, Yang L, Lv X, et al. (2012) HMGB1 mediates IFN$\hat{\mathrm{I}}^{3}$-induced cell proliferation in MMC cells through regulation of cyclin D1/CDK4/p16 pathway. J Cell Biochem 113: 2009-2019.

52. Jin X, Yao T, Zhou Z, Zhu J, Zhang S, et al. (2015) Advanced Glycation End Products Enhance Macrophages Polarization into M1 Phenotype through Activating RAGE/NF-Î०B Pathway. Biomed Res Int 2015: 732450.

53. Deng W, Chen W, Zhang Z, Huang S, Kong W, et al. (2015) Mesenchymal stem cells promote CD206 expression and phagocytic activity of macrophages through IL-6 in systemic lupus erythematosus. Clin Immunol 161: 209-216.

54. Gao S, Mao F, Zhang B, Zhang L, Zhang X, et al. (2014) Mouse bone marrow-derived mesenchymal stem cells induce macrophage M2 polarization through the nuclear factor- $\hat{\imath} \circ \mathrm{B}$ and signal transducer and activator of transcription 3 pathways. Exp Biol Med (Maywood) 239: 366-375.

55. Hu Q, Yang C, Wang Q, Zeng H, Qin W (2015) Demethylzeylasteral (T-96) Treatment Ameliorates Mice Lupus Nephritis Accompanied by Inhibiting Activation of NF-1̂ºB Pathway. PLoS One 10: e0133724. 